\title{
Tyrosine kinase inhibitor therapy-induced changes in humoral immunity in patients with chronic myeloid leukemia
}

Koskela, Hanna L. M.

2017-08

Koskela , H L M , El Missiry , M , Ruusila , A, Koskenvesa , P , Bruemmendorf , T H , Gjertsen , B T , Janssen , J , Lotfi , K, Markevarn , B , Olsson-Stromberg , U , Stenke , L , Stentoft , J , Richter , J , Hjorth-Hansen , H , Kreutzman , A \& Mustjoki , S 2017 , ' Tyrosine kinase inhibitor therapy-induced changes in humoral immunity in patients with chronic myeloid leukemia ' , Journal of Cancer Research and Clinical Oncology , vol. 143 , no. 8 , pp. 1543-1554 . https://doi.org/10.1007/s00432-017-2378-6

http://hdl.handle.net/10138/237301

https://doi.org/10.1007/s00432-017-2378-6

publishedVersion

Downloaded from Helda, University of Helsinki institutional repository.

This is an electronic reprint of the original article.

This reprint may differ from the original in pagination and typographic detail.

Please cite the original version. 


\title{
Tyrosine kinase inhibitor therapy-induced changes in humoral immunity in patients with chronic myeloid leukemia
}

\author{
Hanna L. M. Rajala ${ }^{1}$ Mohamed El Missiry ${ }^{1}$ Anniina Ruusila ${ }^{1} \cdot$ Perttu Koskenvesa $^{1} \cdot$ Tim H. Brümmendorf $^{2}$. \\ Bjorn T. Gjertsen ${ }^{3} \cdot$ Jeroen Janssen $^{4} \cdot$ Kourosh Lotfi $^{5} \cdot$ Berit Markevärn $^{3} \cdot$ Ulla Olsson-Strömberg $^{6} \cdot$ Leif Stenke $^{7}$. \\ Jesper Stentoft ${ }^{8} \cdot$ Johan Richter $^{9} \cdot$ Henrik Hjorth-Hansen ${ }^{10,11} \cdot$ Anna Kreutzman ${ }^{1,12} \cdot$ Satu Mustjoki ${ }^{1,12}$
}

Received: 13 February 2017 / Accepted: 16 February 2017 / Published online: 23 March 2017

(C) Springer-Verlag Berlin Heidelberg 2017

\begin{abstract}
Purpose Tyrosine kinase inhibitors (TKIs) have wellcharacterized immunomodulatory effects on $\mathrm{T}$ and $\mathrm{NK}$ cells, but the effects on the humoral immunity are less well known. In this project, we studied TKI-induced changes in B cell-mediated immunity.

Methods We collected peripheral blood (PB) and bone marrow (BM) samples from chronic myeloid leukemia (CML) patients before and during first-line imatinib $(n=20)$, dasatinib $(n=16)$, nilotinib $(n=8)$, and bosutinib $(n=12)$ treatment. Plasma immunoglobulin levels were measured, and different B cell populations in PB and BM were analyzed with flow cytometry.
\end{abstract}

Electronic supplementary material The online version of this article (doi:10.1007/s00432-017-2378-6) contains supplementary material, which is available to authorized users.

Satu Mustjoki

satu.mustjoki@helsinki.fi

1 Hematology Research Unit Helsinki, Department of Hematology, University of Helsinki and Helsinki University Hospital Comprehensive Cancer Center, Haartmaninkatu 8, 00290 Helsinki, Finland

2 Internal Medicine IV (Oncology, Hematology and Stem Cell Transplantation), University Hospital Aachen (RWTH), Aachen, Germany

3 Institute of Medicine, Hematology Section, University of Bergen, Bergen, Norway

4 Department of Hematology, VU University Medical Center, Amsterdam, Netherlands

5 Department of Hematology and Department of Clinical and Experimental Medicine, Linköping University, Linköping, Sweden

6 Department of Medical Science, Uppsala University Hospital, Uppsala, Sweden
Results Imatinib treatment decreased plasma IgA and IgG levels, while dasatinib reduced IgM levels. At diagnosis, the proportion of patients with $\operatorname{IgA}, \operatorname{IgG}$, and $\operatorname{IgM}$ levels below the lower limit of normal (LLN) was 0,11 , and $6 \%$ of all CML patients, respectively, whereas at 12 months timepoint the proportions were $6 \%(p=0.13), 31 \%$ $(p=0.042)$ and $28 \%(p=0.0078)$. Lower initial Ig levels predisposed to the development of hypogammaglobulinemia during TKI therapy. Decreased Ig levels in imatinibtreated patients were associated with higher percentages of immature BM B cells. The patients, who had low Ig levels during the TKI therapy, had significantly more frequent minor infections during the follow-up compared with the patients with normal Ig values (33\% vs. $3 \%, p=0.0016$ ). No severe infections were reported, except recurrent upper

7 Department of Medicine, Division of Hematology, Karolinska University Hospital and Karolinska Institutet, Stockholm, Sweden

8 Department of Hematology, Aarhus University Hospital, Aarhus, Denmark

9 Department of Hematology, Oncology and Radiation Physics, Skane University Hospital, Lund, Sweden

10 Department of Hematology, St Olavs Hospital, Trondheim, Norway

11 Department of Cancer Research and Molecular Medicine, NTNU, Trondheim, Norway

12 Department of Clinical Chemistry, University of Helsinki, Helsinki, Finland 
respiratory tract infections in one imatinib-treated patient, who developed severe hypogammaglobulinemia.

Conclusions TKI treatment decreases plasma Ig levels, which should be measured in patients with recurrent infections.

Keywords CML - Tyrosine kinase inhibitor - B cell · Immunoglobulin

\section{Introduction}

Chronic myeloid leukemia (CML) originates from a hematopoietic stem cell carrying the Philadelphia $(\mathrm{Ph})$ chromosome and an oncogenic $B C R-A B L 1$ fusion gene, which encodes for a constitutively active tyrosine kinase. Current therapy for CML is based on tyrosine kinase inhibitors (TKIs) imatinib, nilotinib, dasatinib, or bosutinib. The first-generation TKI imatinib has been proved to be both safe and efficient, radically improving the outcome of CML patients (Druker et al. 2006; Hochhaus et al. 2009). The second-generation TKIs nilotinib and dasatinib were originally used as a second-line treatment for patients resistant or intolerant to imatinib treatment. However, as they appear to be more effective than imatinib and generally well tolerated, they are now also being used as a first-line therapy for CML (Kantarjian et al. 2010, 2011). Bosutinib is one of the newest members in the TKI family, and it is used as a second or third line option in CML patients resistant for or intolerant to imatinib, dasatinib, or nilotinib (Khoury et al. 2012).

Although the main target of TKIs is the BCR-ABL1 kinase, they all have a distinct inhibitory profile and affect several other kinases as well (Hantschel et al. 2008). Related to the inhibition of these off-target kinases, all TKIs have potential impact on the immune system and several in vitro studies have suggested mostly immunosuppressive effects (Ilander et al. 2014). In B cells, ABL kinase phosphorylates B cell co-receptor CD19, and thus may regulate the B cell proliferation (Zipfel et al. 2000). Accordingly, ABL knockout mice show severe defects in B and T cell development (Tybulewicz et al. 1991). Neutropenia and thrombocytopenia are common during the first months of the TKI therapy. The exact mechanism of these cytopenias is not known, but the swift clearance of the $\mathrm{Ph}+$ hematopoiesis after the onset of TKI therapy preceding the recovery of normal hematopoiesis may have a role in addition to the direct inhibitory effects of the TKIs (Talpaz et al. 2006; Druker et al. 2006; Quintás-Cardama et al. 2009). Although B cells are Ph-negative in most untreated CML patients, $25 \%$ of the patients have predominantly $\mathrm{Ph}+$ $\mathrm{B}$ cells, or a mixture of $\mathrm{Ph}+$ and $\mathrm{Ph}$-negative $\mathrm{B}$ cells in the bone marrow (Nitta et al. 1985; Takahashi et al. 1998).
Imatinib has been reported to have suppressive effect on B cells with a reduction of IgM+ memory B cells (Lavallade et al. 2013) and, for example, dasatinib inhibits B cell signaling through the Lyn kinase, a member of Src family of tyrosine kinases, directly affecting the B cell compartment (Bantscheff et al. 2007; Rix et al. 2007).

Since CML patients usually need TKI therapy for several years or even decades, the immunological off-target effects may be of significance in clinical practice. Therefore, the aim of this project was to study the effects of TKI therapy on B cell-mediated immunity in vivo in CML patients treated either with first-line imatinib, dasatinib, nilotinib, or bosutinib.

\section{Patients and methods}

\section{Study patients and samples}

Peripheral blood (PB) and bone marrow (BM) samples were collected from newly diagnosed chronic phase CML patients $(n=44)$ treated with imatinib $(n=20)$, dasatinib $(n=16)$, nilotinib $(n=8)$, and bosutinib $(n=12$, only PB samples available). The patients took part in different clinical studies: imatinib- and dasatinib-treated patients participated in the NordCML006 study cohort (ClinicalTrials. gov: NCT00852566), nilotinib patients in the ENEST1st or ENESTnd study (NCT00519090 and NCT00471497, respectively), and bosutinib-treated patients in the BFORE study (NCT02130557). Patient samples and clinical data were obtained from the following centers: Helsinki University Central Hospital (Helsinki, Finland), Skåne University Hospital (Lund, Sweden), Uppsala University Hospital (Uppsala, Sweden), Linköping University Hospital (Linköping, Sweden), Karolinska University Hospital (Stockholm, Sweden), Norrlands University Hospital (Umeå, Sweden), Aarhus University Hospital (Aarhus, Denmark), St Olavs Hospital (Trondheim, Norway), Haukeland University Hospital (Bergen, Norway), and VU University Medical Center (Amsterdam, Netherlands). Control samples were obtained from 19 healthy volunteers, including 7 volunteers from whom BM samples were also taken. The main characteristics of the patients are summarized in Table 1.

\section{Measurement of plasma immunoglobulin levels}

$\mathrm{ACD}$, heparin, or EDTA plasma was separated from PB and stored in $-70^{\circ} \mathrm{C}$. Plasma immunoglobulin (Ig) levels (IgA, IgM, IgG) were measured after thawing with an immunoturbidimetric method in the central laboratory of the Helsinki University Central Hospital (HUSLAB). The Finnish national reference values for the Ig levels were 
Table 1 Clinical characteristics of the CML patients

\begin{tabular}{|c|c|c|c|c|c|c|}
\hline & Imatinib $(n=20)$ & Dasatinib $(n=16)$ & Nilotinib $(n=8)$ & Bosutinib $(n=12)$ & $p$ value & CML, total $(n=56)$ \\
\hline Males/females & $10 / 10$ & $6 / 10$ & $4 / 4$ & $8 / 4$ & 0.51 & $28 / 28$ \\
\hline Age, median (range) & $57(39-77)$ & $49(39-71)$ & $50(47-60)$ & $44(28-77)$ & 0.10 & $53(28-77)$ \\
\hline Sokal score, median (range) & $0.82(0.58-13.22)$ & $0.81(0.61-4.2)$ & $0.8(0.63-1.7)$ & $0.87(0.51-1.68)$ & 0.98 & $0.82(0.51-13.22)$ \\
\hline Total hypogammaglobulinemia ${ }^{a}$ & $7(35 \%)$ & $4(25 \%)$ & $5(63 \%)$ & $3(25 \%)$ & 0.27 & $19(34 \%)$ \\
\hline $\begin{array}{l}\text { HypoIgA at diagnosis, number of } \\
\text { patients }(\%)^{\mathrm{b}}\end{array}$ & $0 / 20(0 \%)$ & $0 / 16(0 \%)$ & $0 / 7(0 \%)$ & $0 / 10(0 \%)$ & NA & $0 / 53(0 \%)$ \\
\hline $\begin{array}{l}\text { HypoIgG at diagnosis, number of } \\
\text { patients }(\%)^{\mathrm{b}}\end{array}$ & $2 / 20(10 \%)$ & $2 / 16(13 \%)$ & $1 / 7(14 \%)$ & $1 / 10(10 \%)$ & 0.99 & $6 / 53(11 \%)$ \\
\hline $\begin{array}{l}\text { HypoIgM at diagnosis, number of } \\
\text { patients }(\%)^{\mathrm{b}}\end{array}$ & $0 / 20(0 \%)$ & $1 / 16(6 \%)$ & $1 / 7(14 \%)$ & $1 / 10(10 \%)$ & 0.47 & $3 / 53(6 \%)$ \\
\hline $\begin{array}{l}\text { HypoIgA at } 12 \text { months, number of } \\
\text { patients }(\%)^{\mathrm{c}}\end{array}$ & $1 / 13(8 \%)$ & $0 / 6(0 \%)$ & $1 / 7(14 \%)$ & $0 / 6(0 \%)$ & 0.66 & $2 / 32(6 \%)$ \\
\hline $\begin{array}{l}\text { HypoIgG at } 12 \text { months, number of } \\
\text { patients }(\%)^{\mathrm{c}}\end{array}$ & $6 / 13(46 \%)$ & $2 / 6(33 \%)$ & $2 / 7(29 \%)$ & $0 / 6(0 \%)$ & 0.25 & $10 / 32(31 \%)$ \\
\hline $\begin{array}{l}\text { HypoIgM at } 12 \text { months, number of } \\
\text { patients }(\%)^{\mathrm{c}}\end{array}$ & $3 / 13(23 \%)$ & $2 / 6(33 \%)$ & $1 / 7(14 \%)$ & $3 / 6(50 \%)$ & 0.51 & $9 / 32(28 \%)$ \\
\hline
\end{tabular}

Statistical analysis: The means of Sokal score and age were compared with one-way ANOVA. Comparisons of categorical variants, i.e., age and hypogammaglobulinemia, were done with Chi-square test. $N A$ not accessible

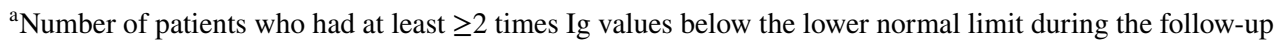

${ }^{\mathrm{b}} \mathrm{Ig}$ levels below the lower limit of normal at diagnosis

${ }^{\mathrm{c}} \mathrm{Ig}$ levels below the lower limit of normal at 12 months timepoint

used in the analysis: $\operatorname{Ig}$ A $0.52-4.84 \mathrm{~g} / \mathrm{l}, \operatorname{IgM} 0.36-2.84 \mathrm{~g} / \mathrm{l}$, IgG $6.8-15 \mathrm{~g} / \mathrm{l}$.

\section{Separation of peripheral blood mononuclear cells}

Mononuclear cells were separated from PB using FicollPaque gradient separation (GE Healthcare, Chicago, Illinois, US) according to the manufacturer's instructions. The isolated PB MNCs were cryopreserved in fetal bovine serum containing $10 \%$ DMSO.

\section{Analysis of PB and BM B cell subclasses by flow cytometry}

PB and BM samples from CML patients treated with imatinib, dasatinib, nilotinib, and bosutinib (only PB) were analyzed at diagnosis and 1, 3, 6, and 12 months after the start of therapy. The samples were stained with antibodies against $\mathrm{CD} 45, \mathrm{CD} 3$, and $\mathrm{CD} 19$. The proportion of CD45+CD19+ B cells was analyzed using flow cytometry after the lysis of red blood cells. In the BM samples, additional B cell populations were gated to analyze different stages of B cell development: CD19+CD45 ${ }^{\text {high }}$ mature, $\mathrm{CD} 19+\mathrm{CD} 45^{\text {intermediate }}$ maturing, and $\mathrm{CD} 19+\mathrm{CD} 45^{\text {low }}$ immature B cells (Caldwell and Patterson 1987; Shah et al. 1988). The flow cytometry analysis was performed with FACSAriaI/II and FACSDiva software (Becton Dickinson, Franklin Lakes, New Jersey, US).
The mature $\mathrm{B}$ cells in $\mathrm{PB}$ were analyzed in more detail at 12 months timepoint by staining $\mathrm{PB}$ mononuclear cells (PB MNCs) with CD3, CD19, CD27, CD38, $\mathrm{IgD}$, and IgM antibodies. B cells were first gated from the lymphocyte population as $\mathrm{CD} 3{ }^{\text {neg }} \mathrm{CD} 19+$ cells, and following subpopulations were gated: $C D 19+C D 27^{\text {neg }}$ mature naïve cells, CD19+CD27+ mature memory cells, CD19+CD27+IgM ${ }^{\text {neg }} \operatorname{IgD}^{\text {neg }}$ class-switched memory cells, CD19+CD27+IgM+IgD ${ }^{+/ l o w}$ marginal zone-like memory cells, CD19+CD38+IgM+ transitional B cells, and CD19+CD $38^{\text {high }} \operatorname{IgM}^{\text {neg }}$ plasmablasts. Samples were analyzed with FACSVerse and FACSDiva or FACSuite softwares (Becton Dickinson).

All antibodies were purchased from Becton Dickinson.

\section{Statistical analysis}

Comparisons of Ig levels and B cell subpopulations were done with Mann-Whitney $t$ test, paired $t$ test, or one- or two-way ANOVA. Clinical correlations were analyzed by Spearman's. Comparisons of categorical variants between groups were done with Chi-square test. $p$ values below 0.05 were considered as statistically significant. All analyses were performed using either GraphPad Prism version 6.0 (GraphPad software Inc., California, US) or SPSS version 22 (IBM SPSS Statistics, Armonk, New York, US) software. 
$\lg A$
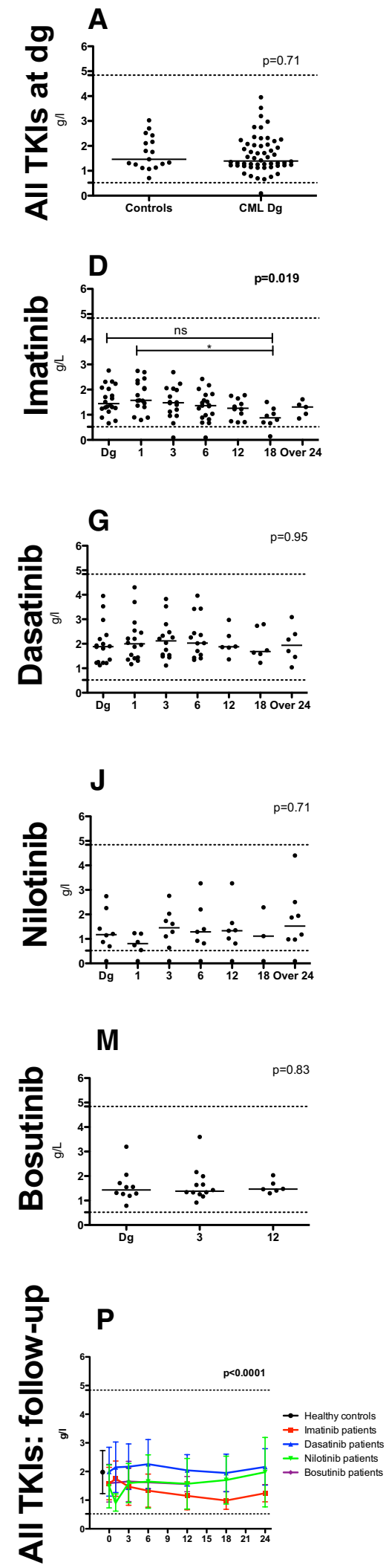

$\lg G$
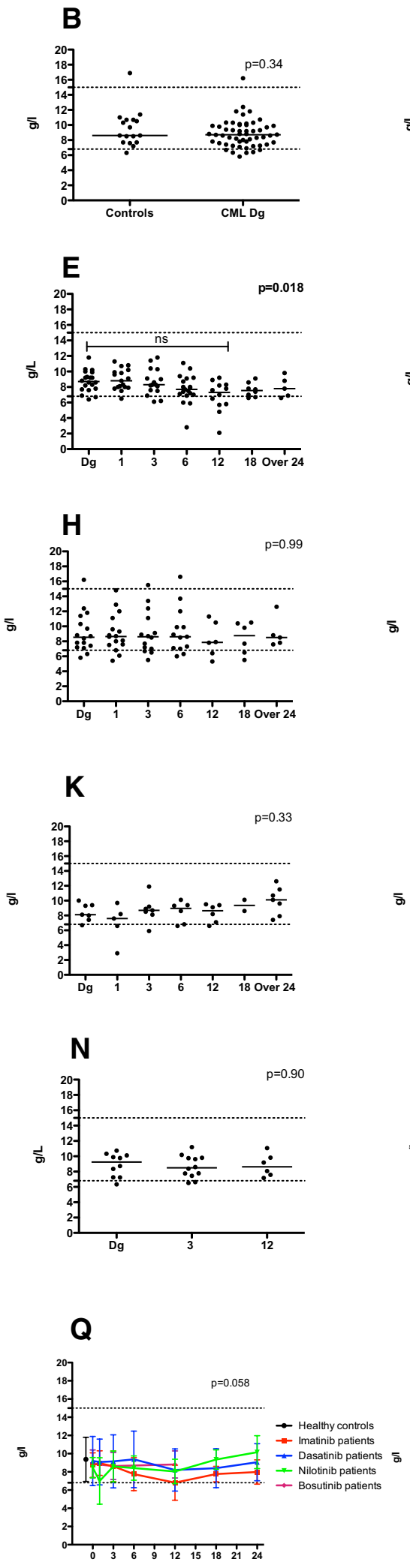

$\lg M$
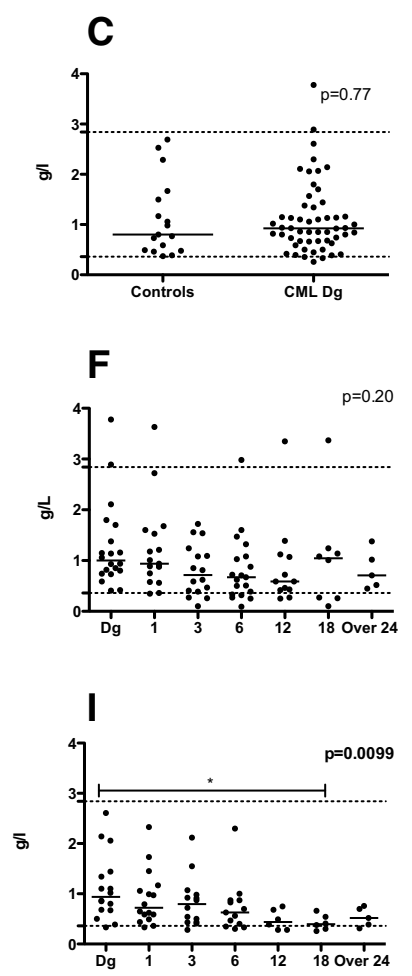

L
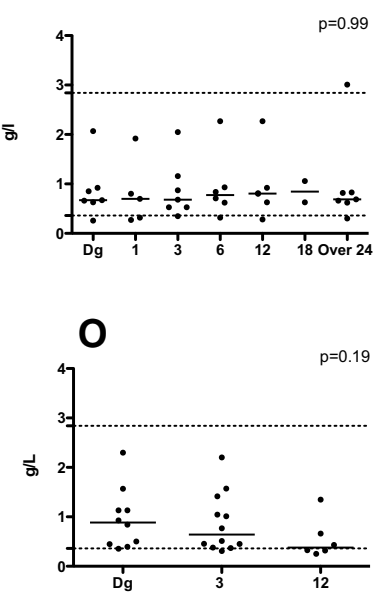

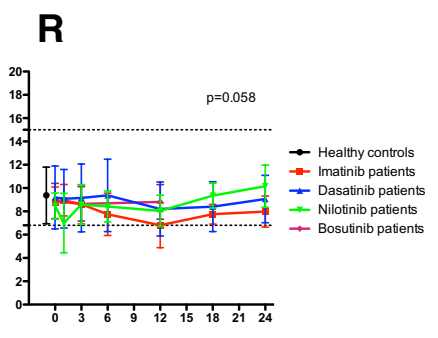


4Fig. 1 Immunoglobulin values of CML patients during follow-up. Dotted lines represent the lower and upper limit of normal Ig values. a-c Diagnostic phase $\operatorname{IgA}(\mathbf{a}), \operatorname{IgG}(\mathbf{b})$, and $\operatorname{IgM}(\mathbf{c})$ values in CML patients were compared with healthy controls. Lines present median values. Mann-Whitney $t$ test was used for statistical analysis. d-o IgA, IgG, and IgM levels in patients treated with imatinib $(\mathbf{d}-\mathbf{f})$, dasatinib $(\mathbf{g}-\mathbf{i})$, nilotinib $(\mathbf{j}-\mathbf{l})$, and bosutinib (m-o) therapy. Lines present median values. One-way ANOVA test was used for statistical analysis. p-r Mean Ig levels of TKI-treated patients at different timepoints. I-bars represent SD. Two-way ANOVA test was used for statistical analysis

\section{Results}

\section{Imatinib and dasatinib treatment are related to lower Ig levels during follow-up}

Plasma Ig levels were analyzed at diagnosis and during TKI therapy $(1,3,6,12,18$, and 24 months after the start of therapy). Plasma samples were available from patients treated with imatinib $(n=20)$, dasatinib $(n=16)$, nilotinib $(n=8)$, or bosutinib $(n=12)$ and 19 healthy controls.

Median Ig levels of chronic phase CML patients were similar to the healthy controls before the onset of TKI treatment (Fig. 1a-c). However, imatinib-treated patients showed a significant overall decrease of IgA and IgG levels during therapy ( $p=0.019$ and $p=0.018$, respectively) (Fig. 1d-f). Dasatinib-treated patients had only decreased IgM levels $(p=0.0099)$ (Fig. 1g-i), and similar trend was also observed in bosutinib group, but no statistically significant changes were observed. During nilotinib therapy, no marked changes in plasma Ig levels were observed (Fig. 1j-1). When all TKIs were compared with each other, only IgA levels differed statistically significantly between the four groups, imatinib-treated patients having the lowest values $(p<0.0001)$ (Fig. 1p-r).

\section{IgG and IgM levels were the lowest at 12 months timepoint}

At diagnosis the proportion of patients with $\operatorname{IgA}, \operatorname{IgG}$, and IgM levels below the lower limit of normal (LLN) was 0,11 , and $6 \%$ of all CML patients, whereas at 12 months timepoint Ig levels were more often below the LLN (6, 31 and $28 \%$, respectively, Table 1 ). The difference was statistically significant in the case of $\operatorname{IgG}(p=0.042)$ and $\operatorname{IgM}$ $(p=0.0078)$. The proportion of patients with low IgA levels did not differ between diagnosis and 12-month analysis $(p=0.13)$.

Next, we performed a paired analysis of Ig levels between the diagnosis and 12-month timepoint (Fig. 2). At the 12-month analysis, imatinib- and dasatinib-treated patients showed significantly lower IgM values. Similar trend was also observed with bosutinib patients, but due to low number of paired samples $(n=5)$, no statistical significance was reached. In IgG levels, marked decrease was observed both in patients treated with imatinib and bosutinib (Fig. 2). Follow-up samples from later timepoints ( $>12$ months) were not available from all patients, and possible correction of Ig levels could not be analyzed.

\section{Lower Ig levels at diagnosis predict hypogammaglobulinemia during therapy}

All CML patients were divided into two groups based on the occurrence of hypogammaglobulinemia ( $\geq 2$ times observed Ig values below the LLN during follow-up) during the TKI therapy (Table 1, Supplementary Figs. 1 and 2 ). The proportion of the patients with Ig levels below the LLN is shown in Table 1 (34\% of all CML patients), and the percentages did not differ significantly between different TKIs (Table 1). The follow-up curves of the separate CML patients are shown in Supplementary Fig. 1. Those patients, who developed hypogammaglobulinemia, had significantly lower $\operatorname{IgG}$ and $\operatorname{IgM}$ levels already at diagnosis, and median Ig levels were significantly lower during follow-up (Fig. 3a-c, Supplementary Fig. 2).

Laboratory parameters and other clinical data were compared between the two groups. Patients with hypogammaglobulinemia had lower white blood cell counts at the diagnosis ( $p=0.021$, Fig. 3d), and no differences were observed in other blood counts, age, or Sokal risk score (data not shown).

\section{TKI treatment affects the $B$ cell population in PB and $B M$ during the follow-up and these changes are related to $\mathrm{Ig}$ levels}

Paired PB and BM samples for flow cytometric analysis were available from imatinib $(n=9)$, dasatinib $(n=6)$ and nilotinib $(n=8)$ - treated patients.

The proportion of PB B cells or the absolute PB B cell count did not significantly differ between healthy controls and CML patients at diagnosis (Fig. 4a, b). When all TKItreated patients were analyzed as one group, both relative and absolute B cell number dropped significantly during TKI therapy ( $p=0.0020$ and $p<0.0001$, respectively) (Fig. 3a, b). All TKIs were associated with similar decrease in the B cell counts, when the patients were analyzed in separate groups (Fig. 4c). The dynamics of developing B cells in BM was analyzed in more detail using increasing CD45 expression as a marker of maturity in the CD19+ $B$ cell population. Imatinib-treated patients, who also showed significant decrease of $\operatorname{IgA}$ and IgG levels during therapy (Figs. 1, 2), had the lowest number of mature CD19+CD45 ${ }^{\text {high }} \mathrm{B}$ cells in BM during follow-up when compared to the dasatinib group (Fig. $4 \mathrm{~d}-\mathrm{g}$ ). The B cell 


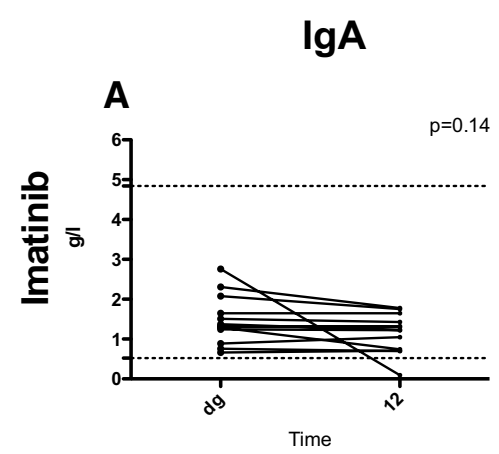

ธิ
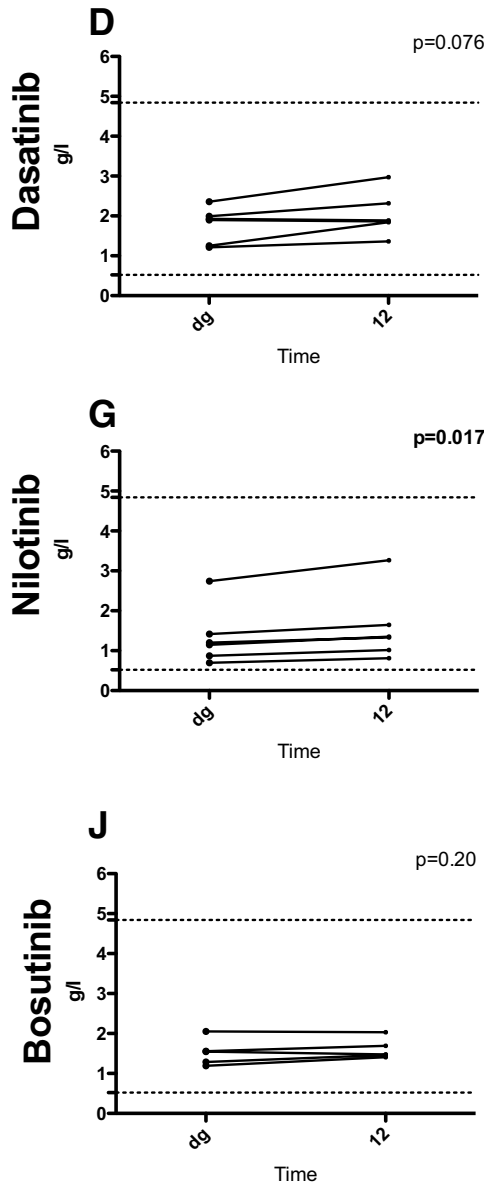

$\lg G$

B

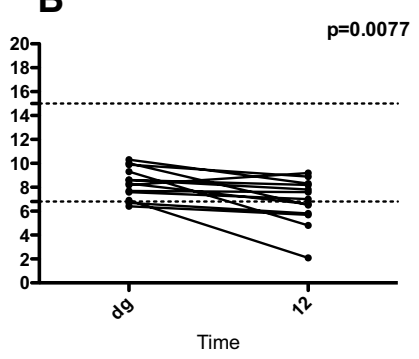

E

$\lg M$

C
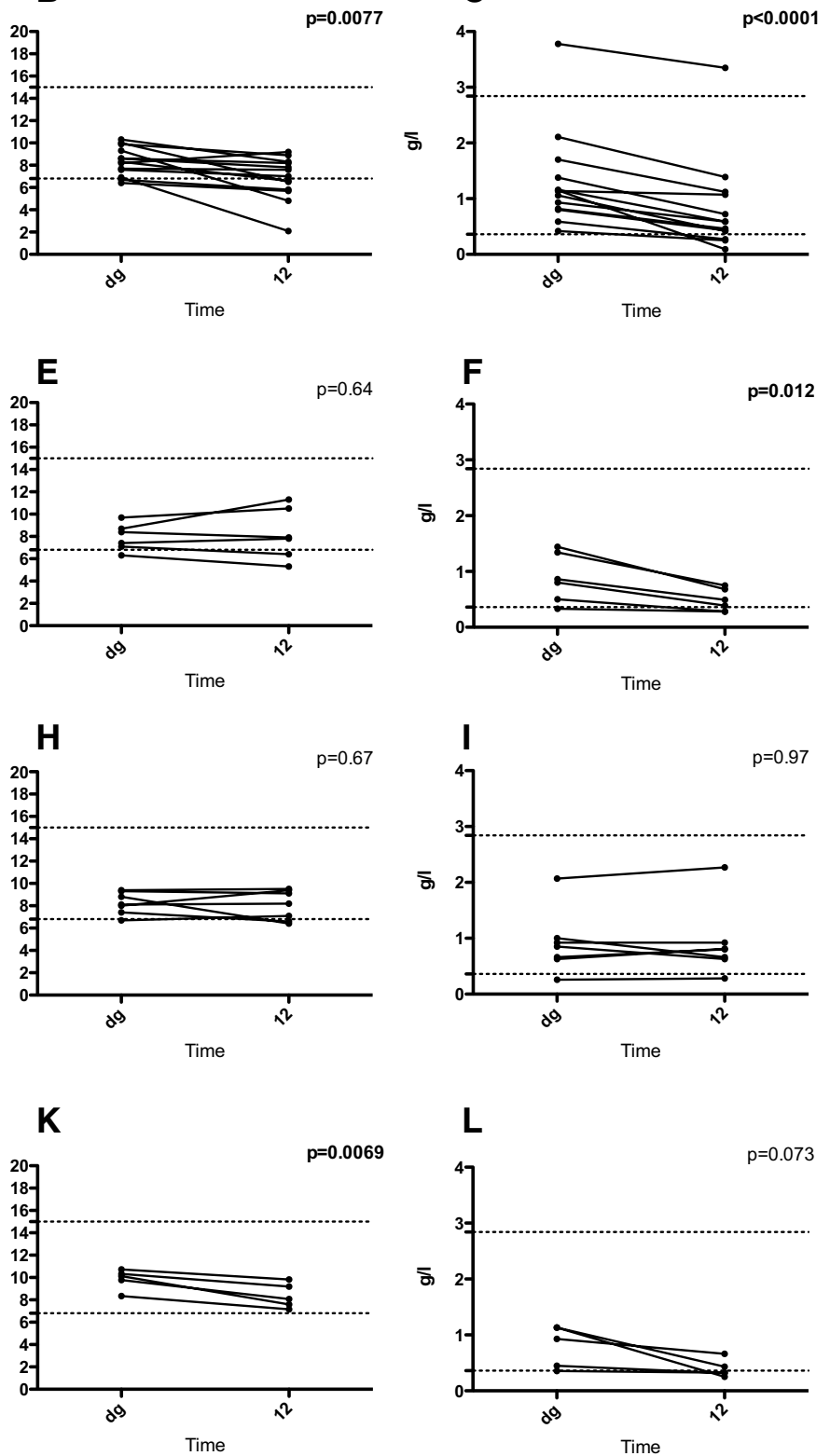

Fig. 2 Paired Ig samples at the diagnosis and after 12 months of TKI therapy. Paired Ig values for diagnostic and 12 month timepoint are shown for imatinib (a-c), dasatinib (d-f), nilotinib (g-i), and bosuti-

profile during nilotinib therapy was similar to that of the imatinib patients (Fig. 4d-f).

\section{PB B cell subpopulations of CML patients did not differ from healthy controls}

We performed a more detailed analysis of the PB B cells at the 12-month timepoint (range 9-18 months). Samples were available from imatinib $(n=11)$, dasatinib $(n=6)$, nib $(\mathbf{j}-\mathbf{l})$-treated CML patients. Dotted lines represent the lower and upper limit of normal Ig values. Paired $t$ test was used for statistical analysis

nilotinib $(n=6)$, or bosutinib-treated $(n=5)$ patients as well as healthy controls $(n=8)$. No significant differences were seen in naïve, memory, and transitional B cells or plasmablasts when CML patients were compared with healthy controls (Fig. 5). Similarly, the occurrence of hypogammaglobulinemia during the follow-up did not affect the PB B cell populations when different TKIs were compared, or when CML patients as one group were compared with the healthy controls (data not shown). During dasatinib 
A

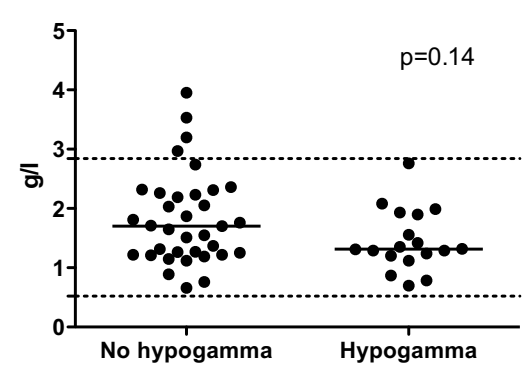

B

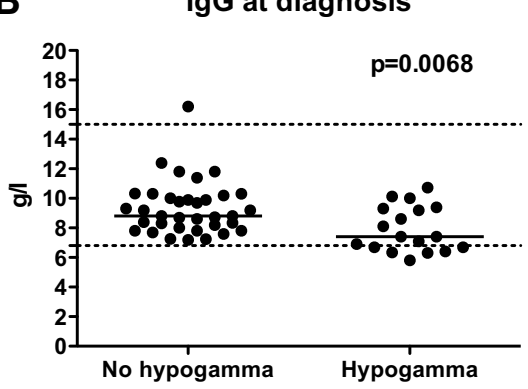

C IgM at diagnosis

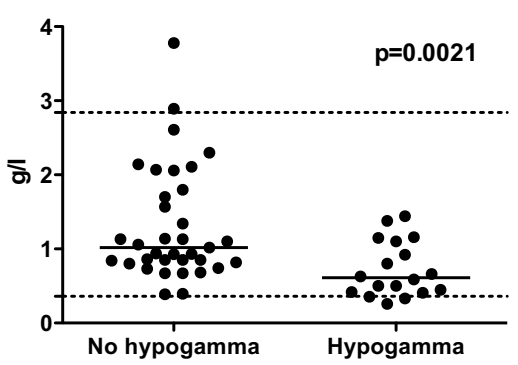

D White blood cells at the diagnosis

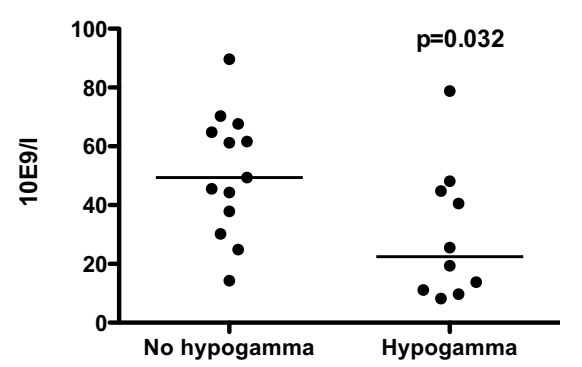

Fig. 3 Lower Ig levels at the diagnosis predicted hypogammaglobulinemia during therapy. CML patients were divided into two groups: the patients who had normal plasma Ig levels during TKI therapy (no hypogamma), and the patients who had $\geq 2$ measured plasma Ig values below the lower limit of normal (hypogamma). a-c Median

treatment, the proportion of memory B cells tended to be larger than in other TKI-treated patients or healthy controls and accordingly the proportion of naïve $\mathrm{B}$ cells was diminished (Fig. 5a, b).

\section{Case report: severe hypogammaglobulinemia during imatinib treatment}

Patient 1 in Table 2 was diagnosed with chronic phase CML at the age of 55 years. The Sokal score was 0.66 (low risk). She used hydroxyurea for 3 weeks, and then started imatinib $400 \mathrm{mg}$ qd. After 3 months of treatment she was in complete cytogenetic remission, and at 12-month timepoint the BCR-ABL qPCR level was $0.38 \%$ IS. The patient suffered from recurrent respiratory tract infections after 9 months of imatinib treatment and was treated with several antibiotics for persistent sinusitis/bronchitis. Ig levels were retrospectively assessed in stored plasma samples. At diagnosis $\operatorname{IgA}, \operatorname{IgG}$, and $\operatorname{IgM}$ levels were within normal range: $2.76,6.9$, and $1.11 \mathrm{~g} / \mathrm{l}$, respectively. The reduction of all Ig levels started at the 3-month timepoint, and at 12 months the patient had developed severe hypogammaglobulinemia (Fig. 6a). The patient switched to dasatinib at 17-month timepoint and started to receive intravenous gammaglobulin diagnostic Ig values were compared between the two groups. d PB leukocyte counts at the diagnosis. Lines represent median values and dotted lines represent the lower and upper limit of normal Ig values. Mann-Whitney $\mathrm{t}$ test was used for statistical analysis

(IVIG). However, during dasatinib therapy the patient developed proctitis and anemia, and had to switch back to imatinib $400 \mathrm{mg}$ OD at 20-month timepoint. Currently, the patient has achieved stable MMR with imatinib. The patient received IVIG every 4-6 weeks for four years, after which the IgG levels were sufficient and IVIG was discontinued. Unusually frequent infections have not been reported since the start of IVIG therapy.

PB counts showed lymphopenia at 1-, 3-, and 6-month timepoints $\left(1.0 \times 10^{9} / 1,0.9 \times 10^{9} / 1\right.$ and $1.1 \times 10^{9} / 1$, respectively) (Fig. 6b). Simultaneously with the decrease of IgM and $\operatorname{IgA}$ levels at 3 months, we observed decreased percentages of BM mature B cells (Fig. 6c) and $\mathrm{PB}$ naïve $\mathrm{B}$ cells (Fig. 6d).

\section{Correlation of immunoglobulin levels with clinical parameters and treatment response}

Ig plasma levels were correlated with clinical parameters and treatment response in the whole CML patient cohort. Higher Sokal score, which is related to the increased CML disease burden, was associated with lower levels of IgM during the follow-up $(p<0.0001$, respectively) 

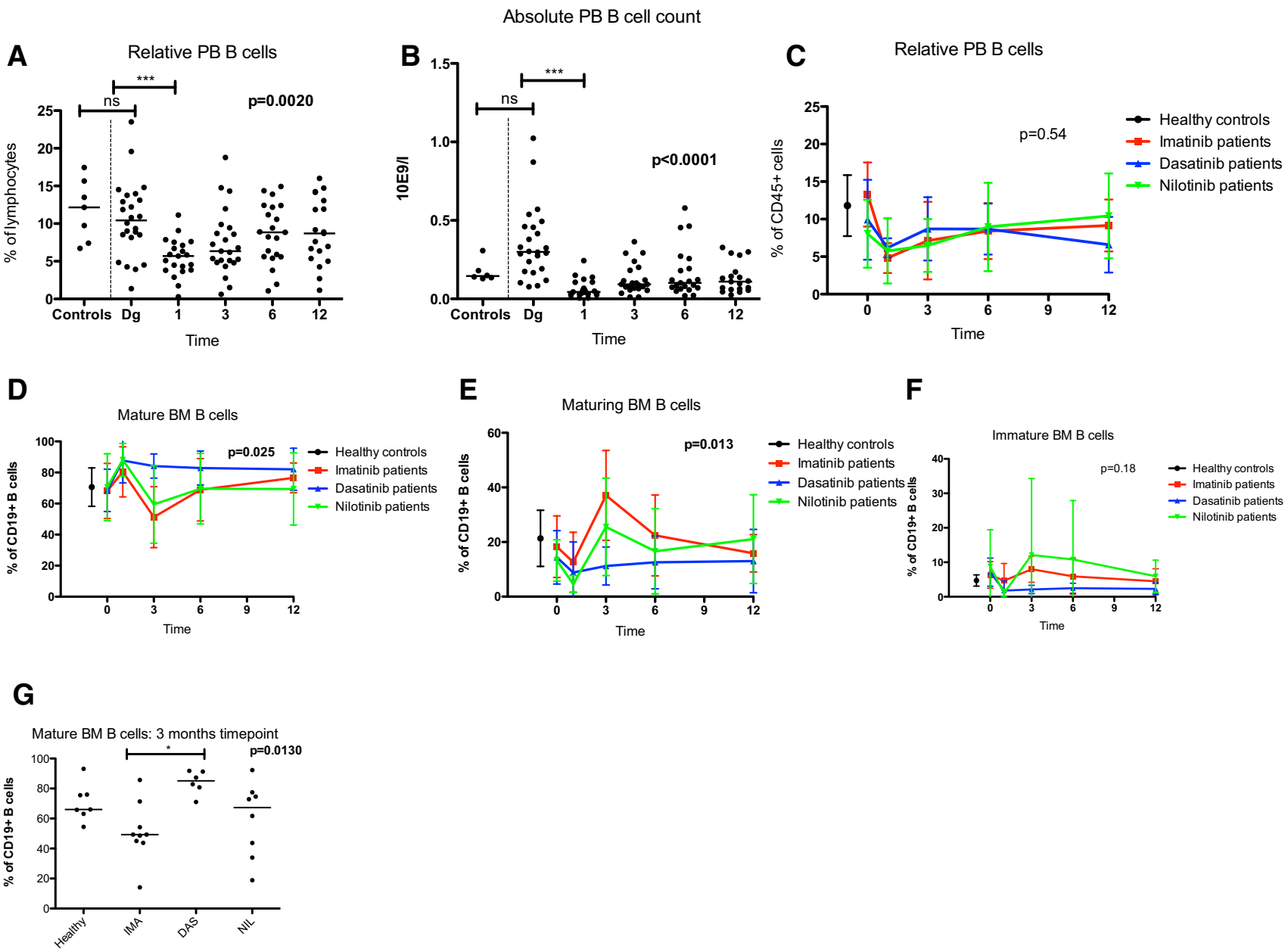

Fig. 4 The analysis of B cell populations in PB and BM during TKI therapy. $\mathbf{a}-\mathbf{b}$ The median proportion of $\mathrm{B}$ cells of CD45+ lymphocytes and absolute number of B cells in healthy controls and all CML patients at different timepoints. One-way ANOVA was used for statistical analysis of CML patients' follow-up data. Mann-Whitney $t$ test was used for the comparison of healthy controls and CML patients at diagnosis, and for the comparison of CML patients' diagnostic phase and 1 month data. $\mathbf{c}$ The comparison of the mean percentage of $\mathrm{PB}$

(Supplementary Fig. 3). Sex or age did not affect Ig levels (data not shown).

Next, we correlated the Ig levels also with treatment response. The achievement of major molecular response [MMR, BCR-ABL $\leq 0.1 \%$ on the International scale (IS)] at 12 months of TKI therapy is a European Leukemia Net criterion for optimal response.(Baccarani et al. 2013) Out of $41 \mathrm{CML}$ patients from whom the data were available, 28 (68\%) had achieved a MMR at 12 months. IgM levels were significantly lower in the patients who had achieved MMR $(p<0.0001)$, but $13 / 28(46 \%)$ of those patients were treated with dasatinib, and accordingly, the observed decrease of IgM values could be due to the overrepresentation of the dasatinib patients (Supplementary Fig. 4, Fig. 1i). The
B cells of CD45+ lymphocytes during different TKI therapies. I-bars represent SD. $\mathbf{d}-\mathbf{f}$ The mean proportions of mature (d), maturing (e), and immature (f) B cells in BM during TKI therapy. I-bars represent SD. $\mathbf{g}$ Median percentages of mature BM B cells of CD19+ B cells at 3 months timepoint. Statistical analysis was done with one-way ANOVA and Bonferroni post-test. Two-way ANOVA was used for the statistical analysis of the data in panels $\mathbf{c}-\mathbf{f}$. $B O S$ bosutinib; $D A S$ dasatinib; IM imatinib; NIL nilotinib. ${ }^{*} p<0.05, * * * p<0.001$

percentages of different B cell populations in PB and BM were not related to the treatment response (data not shown).

\section{Severe infections were not reported in the CML patient cohort}

All studied CML patients took part in different clinical trials and therefore accurate medical documentation including reported infections was available. No major infectious complications were observed among the patients included. One of the nilotinib-treated patients had a primary immunodeficiency syndrome manifesting as a selective IgA-deficiency: the patient was excluded from the analysis regarding $\operatorname{IgA}$ values. Of the 24 patients who had low Ig levels during the 
A

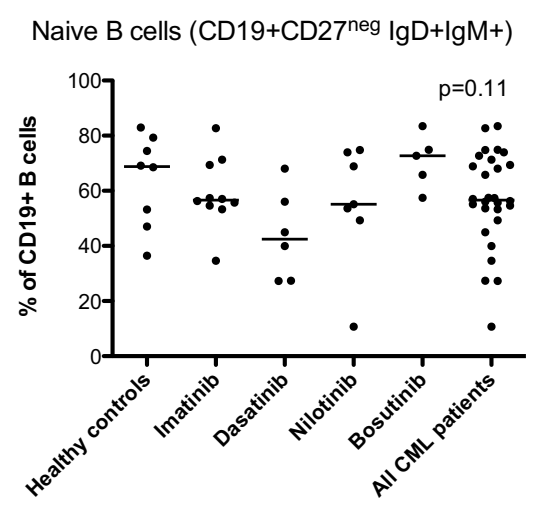

C

Marginal zone like memory B cells $\left(\mathrm{CD} 19+\mathrm{CD} 27+\operatorname{lgM}+\lg \mathrm{D}^{+/ l \text { low }}\right)$

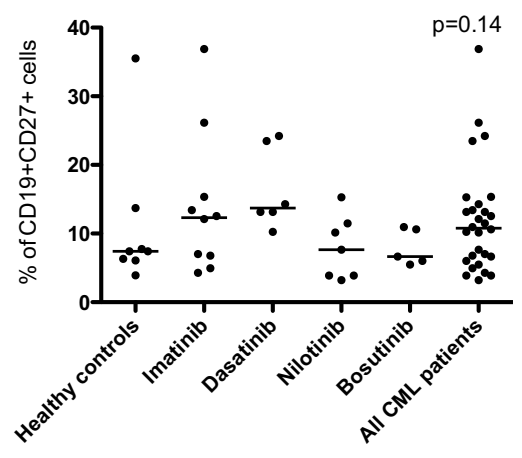

\section{$\mathbf{E}$}

Plasmablasts $\left(C D 19+C D 27+C D 38^{\text {high }} / g^{\text {neg }}\right)$

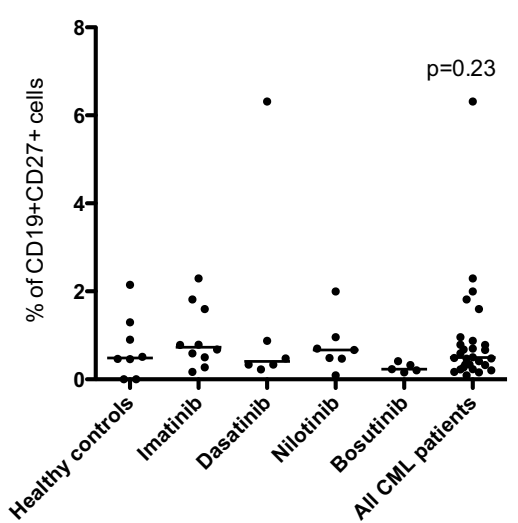

B

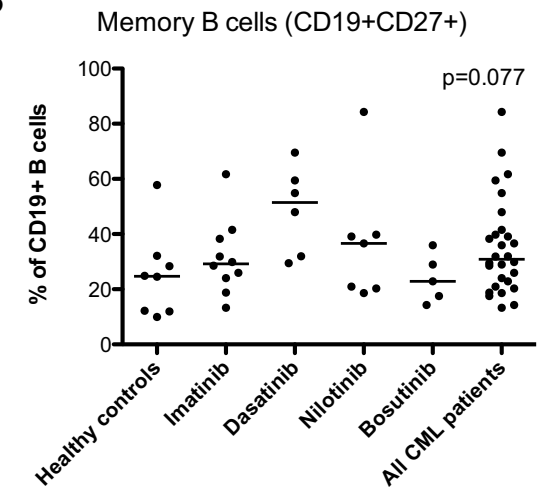

D

Class-switched memory B cells $\left(C D 19+C D 27+\lg M^{\text {neg }} \lg D^{\text {neg }}\right)$

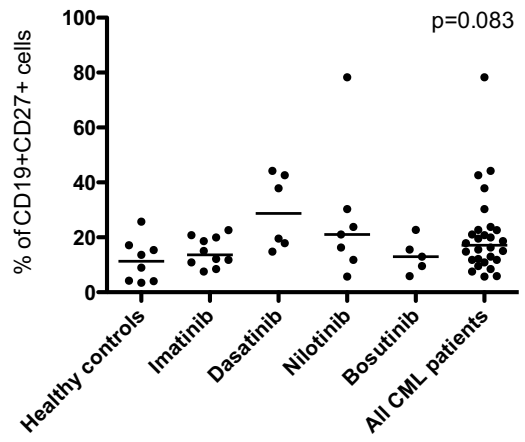

Fig. 5 Flow cytometry analysis of PB B cell subclasses. Following PB B cell subpopulations were analyzed by flow cytometry: Naïve (a), memory (b), marginal zone-like memory (c), class-switched memory (d), and plasmablasts (e). Lines represent median values. One-way ANOVA was used for statistical analysis between the groups 
Table 2 Infections in patients with hypogammaglobulinemia

\begin{tabular}{lll}
\hline Case & Reported infections & Drug \\
\hline $1^{\text {a }}$ & Sinusitis/bronchitis & Imatinib \\
2 & Conjunctivitis, common cold & Imatinib \\
3 & Conjunctivitis & Dasatinib \\
4 & Cystitis caused by E. coli & Dasatinib \\
5 & 2 episodes of common cold, oral herpes simplex & Nilotinib \\
6 & 2 episodes of common cold, oral herpes simplex & Nilotinib \\
7 & Gastroenteritis, a boil in the skin & Nilotinib \\
8 & Common cold & Bosutinib \\
\hline
\end{tabular}

${ }^{\mathrm{a}}$ The patient with severe hypogammaglobulinemia

TKI therapy, minor infections were reported in 8 (33\%) cases during the follow-up, whereas, only 1 (3\%) of the 34 patients with normal Ig levels suffered from minor respiratory tract infections reported in the medical documentation $(p=0.0016)$.

\section{Discussion}

In addition to their main target BCR-ABL1, all available TKIs also inhibit several other kinases, which are important for the function of the immune system (Hantschel et al. 2008). Although immunomodulatory effects of different

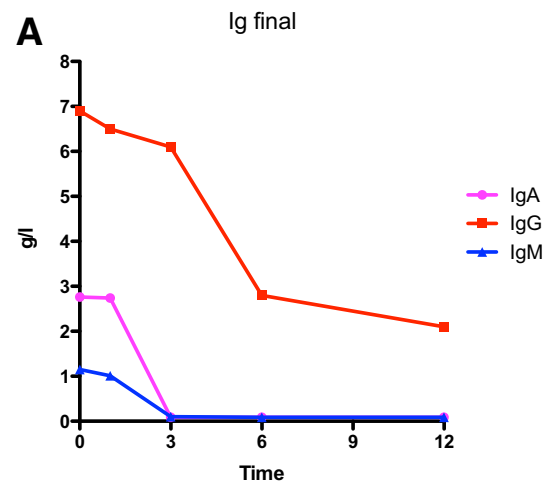

\section{C}

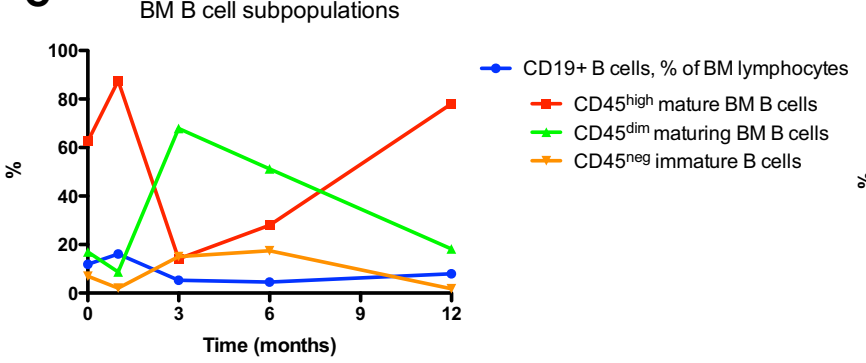

Fig. 6 CML patient with severe hypogammaglobulinemia during imatinib therapy. a IgA, Ig, and $\operatorname{IgM}$ values during the follow-up. b $\mathrm{PB}$ leukocyte counts. c Flow cytometry analysis of BM B cell sub-
TKIs, mainly of imatinib and dasatinib, have previously been described, most of these studies have focused on describing the effects on the innate and adaptive immune systems, thus leaving the humoral immunity less studied. To our knowledge, this is the first study to compare the in vivo effects of four different first-line TKIs (imatinib, dasatinib, nilotinib, bosutinib) on Ig levels and B cell populations in chronic phase CML patients.

The plasma Ig values at diagnosis in our CML patient cohort did not differ from healthy controls, which is in concordance with previous studies (Santachiara et al. 2008). However, lower diagnostic Ig levels predisposed to the development of hypogammaglobulinemia during TKI therapy. In total, 34\% of the CML patients developed hypogammaglobulinemia during first-line TKI therapy, and especially $\operatorname{IgG}$ and $\operatorname{IgM}$ levels decreased when diagnostic values and 12-months analysis were compared. Similar observations have been made before in CML patients resistant to or intolerant of interferon- $\alpha$ and subsequently treated with second-line imatinib (Steegmann et al. 2003). Another study showed, that $42 \%$ of CML and $46 \%$ of gastrointestinal stromal tumor (GIST) patients who used imatinib as a first-line therapy developed hypogammaglobulinemia (Santachiara et al. 2008). The role of TKIs on the humoral immunity has also been studied in the context of vaccines: in a series of CML patients treated with imatinib, dasatinib, or nilotinib and vaccinated against influenza virus, TKI
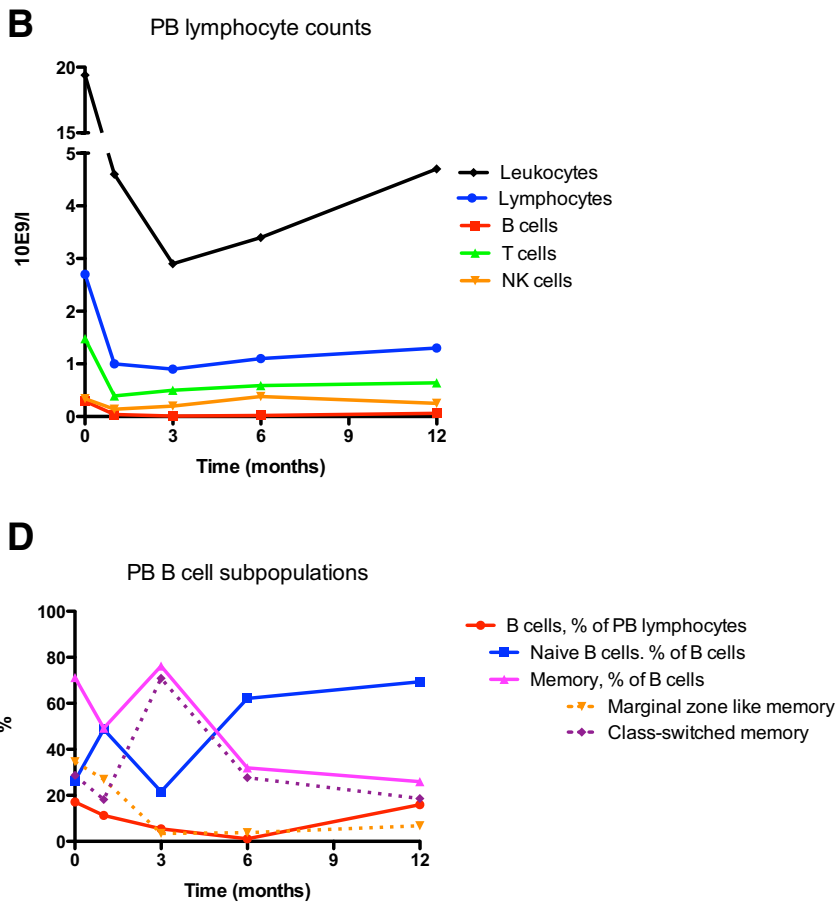

classes. Mature, maturing, and immature B cells were gated from the whole BM B cell population. d Flow cytometry analysis of PB B cell subclasses 
therapy was associated with decreased anti-influenza IgM titers compared to the healthy controls (Lavallade et al. 2013).

In addition to hypogammaglobulinemia, we also observed changes in CML patients' BM and PB B cell populations during the TKI therapy. Imatinib therapy diminished especially the proportion of CD45+ mature B cells in the bone marrow, while dasatinib was associated with the decreased amount of immature B cells. The low amount of mature B cells during imatinib therapy is in well accordance with noted diminished plasma IgG levels as only mature B cells are able to secrete Igs. In another study, imatinib therapy has been shown to alter the phenotype of BM plasma cells in $60 \%$ of the patients (Carulli et al. 2010). In this study, abnormal phenotype was also associated with the reduced serum gammaglobulin levels (Carulli et al. 2010). Imatinib treatment has also been associated with significantly reduced PB memory B cells (Lavallade et al. 2013).

During dasatinib treatment, we observed a significant decrease in plasma IgM levels during the follow-up. The wide inhibition profile of dasatinib includes kinases that are important for B cells, for example BTK and LYN (Hantschel et al. 2008), and therefore the potential of dasatinib has been previously studied in B cell-related disorders like chronic lymphocytic leukemia and mantle cell lymphoma with some promising results (McCaig et al. 2011; Kim et al. 2015). One earlier study showed, that dasatinib reduces the number of human CD19+ peripheral B cells (Oksvold et al. 2015) and inhibits pre-B cells and immature IgM+ B cells in murine model (Duyvestyn et al. 2014). When other second-generation TKIs with wider inhibitory profile than imatinib are considered (Hantschel et al. 2008), bosutinib inhibits similar kinases as dasatinib, but the effects on B cells have not been studied before. There are no previous reports on the effects of nilotinib on B cells either, even though the drug has a similar kinase targeting profile as imatinib (Hantschel et al. 2008). In our patient cohort, the number of the bosutinib- and nilotinib-treated patients was smaller compared to the imatinib and dasatinib groups and the only significant finding in the bosutinib-treated patients was a decrease in IgM levels.

Hypogammaglobulinemia predisposes towards infections that are normally taken care of by antibody responses. Although no severe infections were reported in our study cohort, minor infections were increased among the patients with hypogammaglobulinemia. Previous large clinical trials on the different TKIs have shown some evidence of a slightly increased rate of infections in TKI-treated patients (Reinwald et al. 2016). It should be noted that among 52 patients in our cohort, one imatinib-treated patient developed severe hypogammaglobulinemia and suffered from recurrent respiratory infections. This patient also needed constant immunoglobulin therapy for four years, until Ig levels were sufficient without replacement.

In conclusion, our results indicate that IgM levels decrease in the majority of CML patients during first-line TKI therapy, and one-third of these patients eventually develop hypogammaglobulinemia. Based on our findings, lower Ig values at diagnosis could predict the development of hypogammaglobulinemia. Minor infections were reported more often in the hypogammaglobulinemia group, which should be taken into account during treatment. Larger patient cohorts are warranted in order to confirm these findings.

Acknowledgements The authors thank the personnel at the Hematology Research Unit Helsinki, Helsinki University Hospital HUSLAB flow cytometry laboratory, and Aija Helin from HUSLAB for their expert technical assistance. This work was supported by the Academy of Finland, the Finnish Cancer Societies, the Sigrid Juselius Foundation, the Finnish Cancer Institute, the Signe and Ane Gyllenberg Foundation, the Otto A. Malm Foundation, the EUTOS project for CML 2016, and investigator initiated research grants from Pfizer, and Novartis. NordCML Study Group has received research funding from Bristol-Myers Squibb for the NordCML006 and NordCML007 clinical studies.

Funding This work was supported by the Academy of Finland, the Finnish Cancer Societies, the Sigrid Juselius Foundation, the Finnish Cancer Institute, the Signe and Ane Gyllenberg Foundation, the Otto A. Malm Foundation, the EUTOS project for CML 2016, and investigator initiated research grants from Pfizer and Novartis. NordCML Study Group has received research funding from Bristol-Myers Squibb for the NordCML006 and NordCML007 clinical studies.

\section{Compliance with ethical standards}

Conflict of interest $\mathrm{SM}, \mathrm{JR}$, and $\mathrm{HHjH}$ have obtained honoraria and research funding from Bristol-Myers Squibb, Novartis, Ariad, and Pfizer.

Ethical approval All procedures performed in studies involving human participants were in accordance with the ethical standards of the institutional and national research committee and with the 1964 Helsinki declaration and its later amendments.

Informed consent Informed consent was obtained from all individual participants included in the study.

\section{References}

Baccarani M, Deininger MW, Rosti G et al (2013) European LeukemiaNet recommendations for the management of chronic myeloid leukemia: 2013. Blood 122:872-884. doi:10.1182/ blood-2013-05-501569

Bantscheff M, Eberhard D, Abraham Y et al (2007) Quantitative chemical proteomics reveals mechanisms of action of 
clinical ABL kinase inhibitors. Nat Biotechnol 25:1035-1044. doi:10.1038/nbt1328

Caldwell CW, Patterson WP (1987) Relationship between T200 antigen expression and stages of B cell differentiation in resurgent hyperplasia of bone marrow. Blood 70:1165-1172

Carulli G, Cannizzo E, Ottaviano V et al (2010) Abnormal phenotype of bone marrow plasma cells in patients with chronic myeloid leukemia undergoing therapy with Imatinib. Leuk Res 34:13361339. doi:10.1016/j.leukres.2010.01.012

Druker BJ, Guilhot F, O'Brien SG et al (2006) Five-year follow-up of patients receiving Imatinib for chronic myeloid leukemia. N Engl J Med 355:2408-2417. doi:10.1056/NEJMoa062867

Duyvestyn JM, Taylor SJ, Dagger SA, et al (2014) Dasatinib targets B-lineage cells but does not provide an effective therapy for myeloproliferative disease in c-Cbl RING finger mutant mice. PLoS ONE 9:e94717. doi:10.1371/journal.pone.0094717

Hantschel O, Rix U, Superti-Furga G (2008) Target spectrum of the BCR-ABL inhibitors imatinib, nilotinib and dasatinib. Leuk Lymphoma 49:615-619. doi:10.1080/10428190801896103

Hochhaus A, O'Brien SG, Guilhot F et al (2009) Six-year follow-up of patients receiving imatinib for the first-line treatment of chronic myeloid leukemia. Leukemia 23:1054-1061. doi:10.1038/leu.2009.38

Ilander M, Hekim C, Mustjoki S (2014) Immunology and immunotherapy of chronic myeloid leukemia. Curr Hematol Malig Rep 9:17-23. doi:10.1007/s11899-013-0190-1

Kantarjian H, Shah NP, Hochhaus A et al (2010) Dasatinib versus Imatinib in newly diagnosed chronic-phase chronic myeloid leukemia. N Engl J Med 362:2260-2270. doi:10.1056/ NEJMoa1002315

Kantarjian HM, Giles FJ, Bhalla KN et al (2011) Nilotinib is effective in patients with chronic myeloid leukemia in chronic phase after imatinib resistance or intolerance: 24-month follow-up results. Blood 117:1141-1145. doi:10.1182/blood-2010-03-277152

Khoury HJ, Cortes JE, Kantarjian HM et al (2012) Bosutinib is active in chronic phase chronic myeloid leukemia after imatinib and dasatinib and/or nilotinib therapy failure. Blood 119:3403-3412. doi:10.1182/blood-2011-11-390120

Kim A, Seong KM, Kang HJ et al (2015) Inhibition of Lyn is a promising treatment for mantle cell lymphoma with bortezomib resistance. Oncotarget 6:38225-38238. doi:10.18632/oncotarget.5425

Lavallade H de, Khoder A, Hart M et al (2013) Tyrosine kinase inhibitors impair B-cell immune responses in CML through offtarget inhibition of kinases important for cell signaling. Blood 122:227-238. doi:10.1182/blood-2012-11-465039

McCaig AM, Cosimo E, Leach MT, Michie AM (2011) Dasatinib inhibits B cell receptor signalling in chronic lymphocytic leukaemia but novel combination approaches are required to overcome additional pro-survival microenvironmental signals. Br J Haematol 153:199-211. doi:10.1111/j.1365-2141.2010.08507.x
Nitta M, Kato Y, Strife A et al (1985) Incidence of involvement of the $\mathrm{B}$ and $\mathrm{T}$ lymphocyte lineages in chronic myelogenous leukemia. Blood 66:1053-1061

Oksvold MP, Duyvestyn JM, Dagger SA et al (2015) The targeting of human and mouse B lymphocytes by dasatinib. Exp Hematol 43(352-363):e4. doi:10.1016/j.exphem.2015.01.008

Quintás-Cardama A, De Souza Santos FP, Kantarjian H et al (2009) Dynamics and management of cytopenias associated with dasatinib therapy in patients with chronic myeloid leukemia in chronic phase after Imatinib failure. Cancer 115:3935-3943. doi: $10.1002 /$ cncr.24432

Reinwald M, Boch T, Hofmann W-K, Buchheidt D (2016) Risk of infectious complications in hemato-oncological patients treated with kinase inhibitors. Biomark Insights 10:55-68. doi:10.4137/ BMI.S22430

Rix U, Hantschel O, Dürnberger G et al (2007) Chemical proteomic profiles of the BCR-ABL inhibitors imatinib, nilotinib, and dasatinib reveal novel kinase and nonkinase targets. Blood 110:4055-4063. doi:10.1182/blood-2007-07-102061

Santachiara R, Maffei R, Martinelli S et al (2008) Development of hypogammaglobulinemia in patients treated with imatinib for chronic myeloid leukemia or gastrointestinal stromal tumor. Haematologica 93:1252-1255. doi:10.3324/haematol.12642

Shah VO, Civin CI, Loken MR (1988) Flow cytometric analysis of human bone marrow. IV. Differential quantitative expression of T-200 common leukocyte antigen during normal hemopoiesis. J Immunol 140:1861-1867

Steegmann JL, Moreno G, Alaez C et al (2003) Chronic myeloid leukemia patients resistant to or intolerant of interferon alpha and subsequently treated with imatinib show reduced immunoglobulin levels and hypogammaglobulinemia. Haematologica $88: 762-768$

Takahashi N, Miura I, Saitoh K, Miura AB (1998) Lineage involvement of stem cells bearing the philadelphia chromosome in chronic myeloid leukemia in the chronic phase as shown by a combination of fluorescence-activated cell sorting and fluorescence in situ hybridization. Blood 92:4758-4763

Talpaz M, Shah NP, Kantarjian H et al (2006) Dasatinib in Imatinibresistant philadelphia chromosome-positive leukemias. N Engl J Med 354:2531-2541. doi:10.1056/NEJMoa055229

Tybulewicz VLJ, Crawford CE, Jackson PK et al (1991) Neonatal lethality and lymphopenia in mice with a homozygous disruption of the c-abl proto-oncogene. Cell 65:1153-1163. doi:10.1016/0092-8674(91)90011-M

Zipfel PA, Grove M, Blackburn K et al (2000) The c-Abl Tyrosine Kinase Is Regulated Downstream of the B Cell Antigen Receptor and Interacts with CD19. J Immunol 165:6872-6879. doi:10.4049/jimmunol.165.12.6872 